\title{
TUBERCULOSIS IN PREGNANCY
} Dr. Sathiyakala Rajendiran, Associate Professor
Dr. Suthanthiradevi, Professor \& Head

Department of Obstetrics \& Gynecology, Shri Sathya Sai Medical College and Research Institute, Sri Balaii Vidyapeeth, Nellikuppam, Kancheepuram Dist., Tamil Nadu - 603 108, India.

\section{Introduction}

Tuberculosis is a common air-borne infection which affects one-third of world's population. There are 8 million new cases and 2 million deaths every year due to tuberculosis as per estimates. $^{(1)}$

India accounts for $30 \%$ of the burden of all tuberculous cases in the world.2 $90 \%$ of the infected patients remain dormant and only $10 \%$ of the infected patients exhibit active tuberculosis.

\section{Tuberculosis and pregnancy}

As tuberculosis in India is more prevalent in the general population, it is not uncommon to find pregnant women being infected by tuberculosis .Incidence and mode of transmission of tuberculosis are not altered by pregnancy. Pregnancy doesn't alter the clinical course of tuberculosis. Dormant infection during pregnancy doesn't cause any major maternal or fetal adverse outcomes.

Active infection results in preterm delivery, low birth weight, intrauterine growth restriction and increase in perinatal mortality. The outcome of pregnancy depends on the site of infection and timing of diagnosis in relation to delivery.

Extra pulmonary tuberculosis accounts for $20 \%$ of the tuberculosis infection. ${ }^{(3)}$ During pregnancy, extra pulmonary site infections such as renal, intestinal, skeletal and meningeal tuberculosis has been reported. ${ }^{(4)}$

Congenital tuberculosis is a rare complication of in-utero tuberculous infection due to maternal hematogenous spread. Only 300 cases have been reported in literature. ${ }^{(5)}$

\section{Screening tests}

Tuberculin skin test and interferon gamma release assays are the two screening tests for detecting tuberculosis, available at present.

Tuberculin skin test: In this test, purified protein derivative (PPD) of intermediate strength -5 tuberculin units, is used. If intracutaneous administration of PPD results in induration of $\geq 5 \mathrm{~mm}$, it is considered positive and further evaluation with chest radiograph (with lead shielding of abdomen) is needed. If the induration is $\geq 10 \mathrm{~mm}$, no further evaluation is needed and treatment should be initiated. HIV testing should be offered to all tuberculin skin test positive patients.

In India, as the prevalence of tuberculosis is high, more than $50 \%$ of the Indian population show tuberculin skin test positive. Hence, tuberculin skin test is of limited value for screening in India. ${ }^{(6)}$ Pregnancy doesn't affect the response to tuberculin skin test. ${ }^{(7)}$ But in immunocompromised patients such as HIV infected patients, induration of $\geq 5 \mathrm{~mm}$ should be considered an indication for treatment without further evaluation.

Interferon Gamma Release Assays (IGRAs): IGRA is a blood test which measures interferon gamma release in response to antigens present in mycobacterium tuberculosis but not Bacille-Calmette Gue'rin(BCG). The two IGRAs available are QuantiFERON -TB Gold and T -SPOT. IGRAs are highly reliable in diagnosing tuberculosis especially during pregnancy. ${ }^{(8)}$

\section{Diagnosis}

The symptoms of active tuberculosis in pregnancy include low grade fever, cough with minimal sputum, hemoptysis and weight loss. Chest radiograph shows infiltrative patterns, 
cavitation or mediastinal lymphadenopathy. Stained smears of sputum shows acid fast bacilli. Sputum AFB culture can also be done to confirm the diagnosis. Nucleic acid amplification assay and drug sensitivity testing can also be done.

\section{Isoniazid (INH) prophylaxis}

The indications for giving INH prophylaxis during pregnancy include tuberculin skin test positive, HIV positive or recent tuberculosis exposure. Pyridoxine in the dosage of 25 to $50 \mathrm{mg} /$ day, should be given along with INH, to prevent neuropathy.

\section{Safety profile of antitubercular drugs ${ }^{(9)}$}

Though Isoniazid (H), Rifampicin(R) and Ethambutol (E) cross the placenta, they do not cause teratogenicity in fetus. Isoniazid and Ethambutol belong to pregnancy category A drugs. Rifampicin belongs to pregnancy category $C$ drugs. Pyrazinamide $(\mathrm{Z})$ is pregnancy category B2 drug. Pyrazinamide is gaining popularity for use during pregnancy and is recommended by WHO and International Union Against Tuberculosis and Lung disease. Hence, all the four drugs (HRZE) can be safely used during pregnancy.

Drugs which are proved to be teratogenic, and hence contraindicated during pregnancy include Streptomycin, Kanamycin, Amikacin, Capreomycin and Fluoroquinolones.

\section{Treatment during pregnancy}

In active tuberculosis cases, the four drug regimen (HRZE + pyridoxine )is recommended. In the first two months, the bactericidal phase, HRZE is given. In the next four months, the continuation phase, HR is given. ${ }^{(8)}$

Liver function test is done every month to rule out hepatic dysfunction due to anti tubercular treatment. There are no specific recommendations for labour and delivery except infection precautions for active disease.

\section{Treatment in postpartum period}

There is increased risk for hepatic dysfunction in the postpartum period if the mother is on anti tubercular treatment. Hence strict surveillance of liver function test is recommended. Isolation of baby from the mother is not needed but contact should be limited. The mother should wear a surgical mask until she becomes smear negative. Breast feeding is not contraindicated. The prognosis is bad if tuberculosis is diagnosed at late stage of the disease in the postpartum period.

If there is no clinical suspicion of congenital tuberculosis, after obtaining gastric aspirate from the infant, INH prophylaxis $(10 \mathrm{mg} / \mathrm{kg} /$ day $)$ with pyridoxine $(5 \mathrm{mg}$ daily) should be commenced and continued for three months. If the tuberculin skin test is negative at three months, BCG vaccine should be given. In case of positive tuberculin test in the baby, INH prophylaxis is given for six months after ruling out active tuberculosis.Even though antitubercular drugs are secreted in breast milk, the concentration is so low that neither therapeutic nor toxic level is reached in the baby. Hence, appropriate anti tubercular treatment must be given for baby with active disease.

\section{Multi drug resistant tuberculosis (MDR-TB)}

Though it's rare, the morbidity due to MDR-TB is high. There are no existing guidelines for the treatment of MDR-TB and hence, elective abortion should be considered. ${ }^{(8)}$ If the patient wants to continue the pregnancy, counseling should be given regarding the risks of exposing the fetus to the known and unknown side effects of second line drugs. Breast feeding is contraindicated. Isolation of baby from mother should be done until she is smear negative.

\section{Concurrent HIV and tuberculosis infection}

The prognosis is poor for both mother and baby if there is concurrent HIV and tuberculosis infection. The risk of transmission of both infection to the infant is increased. Treatment of these women is really a challenging one. Beginning concomitant therapy with anti-retroviral and anti-tubercular drugs can cause immune reconstitution inflammatory syndrome (IRIS) with toxic drug effects. ${ }^{10}$ Recent studies support earlier administration of highly active anti-retroviral therapy(HAART) -within 2 to 4 weeks- after starting antituberculous drugs. ${ }^{(11,12,13)}$

\section{Prevention}

BCG vaccination in childhood, isolation of open TB cases and their prompt treatment and screening of all close contacts of tuberculosis-affected patients can prevent the morbidity and mortality associated with tuberculosis in pregnancy. These strategies can also reduce the overall tuberculous disease burden in the world. 


\section{REFERENCES}

1. Dye $\mathrm{C}$, Scheele S, Dolin P, et al.Consensus statement. Global burden of tuberculosis: estimated incidence, prevalence, and mortality by country. WHO Global Surveillance and Monitoring Project. JAMA Aug 1999; 282(7):677-86.

2. World Health Organisation; Research for Action :understanding and controlling tuberculosis in India; 2000:12.

3. Wilson E. Thelin T, Dilts P. Tuberculosis complicated by pregnancy. Am J Obstet Gynaecol 1972; 115:526-31.

4. Jana N, Vasishta K, Saha SC, Ghosh K.Obstetrical outcomes among women with extrapulmonary tuberculosis.N Engl J Med. Aug 1999; 341(9):645-9.

5. Armstrong L, Garay SM. Tuberculosis and pregnancy and tuberculous mastitis. In: Rom WN, Garay SM eds Tuberculosis. Boston. Little Brown and Company; 96:89-98.

6. Bhide A, Arulkumaran S, Damania KR, Daftary SN. Arias' Practical Guide to High Risk Pregnancy and Delivery. A South Asian perspective. 4th edition. 328-330.
7. Present $P$, Comstock GW. Tuberculin sensitivity in pregnancy. Am Rev Respir Dis $1975 ; 112: 413-16$.

8. Cunningham, Leveno, Bloom et al. Williams obstetrics. 24th edition.1019-1021.

9. Guideline -Treatment of tuberculosis in pregnant women and newborn infants. version 3. Department of health-Queensland.

10. Török ME, Yen NT, Chau TT et al.Timing of initiation of antiretroviral therapy in human immunodeficiency virus (HIV) associated tuberculous meningitis. Clin Infect Dis. 2011 Jun; 52(11):1374-83.

11. Blanc FX, Sok T, Laureillard D et al. CAMELIA Study Team. Earlier versus later start of antiretroviral therapy in HIV-infected adults with tuberculosis. N Engl J Med. 2011 Oct 20; 365(16):1471-81.

12. Havlir DV, Kendall MA, Ive P, Kumwenda J et al. AIDS Clinical Trials Group Study A5221. Timing of antiretroviral therapy for HIV-1 infection and tuberculosis. N Engl J Med. 2011 Oct 20; 365(16):1482-91.

13. Karim $A$, Naidoo $K$, Grobler $A$ et al. Integration of Antiretroviral Therapy with Tuberculosis Treatment. N Engl J Med. 2011 0ct 20; 365(16):1492-1501. 